\title{
Treatment Patterns, Depression, and Anxiety Among US Patients Diagnosed with Hyperhidrosis: A Retrospective Cohort Study
}

\author{
Stephanie Z. Klein · Michael Hull · Kristin Khalaf Gillard • \\ Jesse Peterson-Brandt
}

Received: July 13, 2020 / Published online: September 11, 2020

(C) The Author(s) 2020

\section{ABSTRACT}

Introduction: Hyperhidrosis is associated with social and emotional stress due to limitations on health-related quality of life. This study examined real-world treatment patterns and concomitant depression and/or anxiety in patients with hyperhidrosis.

Methods: Commercial health plan members in the US with $\geq 2$ hyperhidrosis diagnosis codes and/or antiperspirant prescription claims were identified from January 2010 through November 2017. A control cohort (CC) of patients without hyperhidrosis was matched to the

Digital Features: To view digital features for this article go to https://doi.org/10.6084/m9.figshare.12821228.

Electronic supplementary material The online version of this article (https://doi.org/10.1007/s13555020-00439-y) contains supplementary material, which is available to authorized users.

\section{S. Z. Klein}

Department of Dermatology, University of Utah, 30 North 1900 East, 4A330, Salt Lake City, UT 84132, USA

M. Hull · J. Peterson-Brandt

Optum Life Sciences, 11000 Optum Circle, Eden Prairie, MN 55344, USA

K. K. Gillard $(\bowtie)$

Dermira, Inc., a wholly-owned subsidiary of Eli Lilly and Company, 275 Middlefield Road, Suite 150, Menlo Park, CA 94025, USA

e-mail: kristin.gillard@dermira.com hyperhidrosis cohort on demographic characteristics. Depression and/or anxiety were identified by $\geq 1$ relevant diagnosis code or pharmacy claim. A multivariable logistic regression model estimated odds of treatment in the hyperhidrosis cohort, and depression/ anxiety in the hyperhidrosis cohort and CC, adjusting for patient characteristics.

Results: A total of 44,484 patients with hyperhidrosis were identified, of whom $58.5 \%$ were female, with a mean ( \pm standard deviation) age of $36.5 \pm 16.5$ years $(83.5 \% \geq 18$ years $)$. A small majority of patients $(51.6 \%, 0.69 /$ person-year $)$ received treatment with prescription antiperspirants. Post-index oral systemic therapies, medical procedures, and surgical options were uncommon. At 12 months post-index, $48.4 \%$ of the sample had not filled a prescription for extra- or prescription-strength antiperspirants. Compared with the CC $(n=137,451)$, a higher percentage of patients with hyperhidrosis had depression or anxiety reported during follow-up (41.1 vs. $28.2 \%, p<0.001$ ); this corresponded to higher adjusted odds of depression/anxiety in patients with hyperhidrosis [odds ratio (OR) 1.76, 95\% confidence interval (CI) $1.72-1.80, p<0.001]$. Baseline depression and/ or anxiety were associated with lower odds of receiving hyperhidrosis treatment (OR 0.77, 95\% CI 0.73-0.80), as was increasing age and male gender. Patients with hyperhidrosis also had more frequent incident depression/anxiety during follow-up (18.2 vs. 10.6\%, $p<0.001)$. 
Conclusion: In this real-world analysis, hyperhidrosis was associated with increased odds of depression and/or anxiety. However, relatively low percentages of patients received prescription topical or oral treatments or underwent surgery, suggesting that tolerability, efficacy, and provider awareness may be limiting factors in the effective treatment of hyperhidrosis.

Keywords: Anxiety; Depression; Hyperhidrosis

\section{Key Summary Points}

The prevalence of hyperhidrosis in the USA is estimated to be $4.8 \%$; however, patients may be underdiagnosed, undertreated, and may not seek treatment for symptom relief.

Hyperhidrosis is associated with social and emotional distress, yet rates of diagnosed anxiety and depression have not been evaluated among a large, nationally representative cohort of hyperhidrosis patients.

In this study we evaluated treatment rates and patterns among individuals in the USA diagnosed with hyperhidrosis and estimated the incidence and prevalence of depression and anxiety among these patients.

Many of these patients with hyperhidrosis did not receive treatment for their condition, and compared to matched controls, patients with hyperhidrosis had higher adjusted odds of depression and/or anxiety (odds ratio $1.76,95 \%$ confidence interval 1.72-1.80, $p<0.001$ ).

Hyperhidrosis may have a considerable impact on the quality of life and mental health which may be comparable to that associated with other chronic and impactful conditions.
Providers who treat hyperhidrosis should recognize the potential for comorbid anxiety and depression, and screening for these conditions and linking patients with mental health resources may help to improve patients' overall health and wellbeing.

\section{DIGITAL FEATURES}

This article is published with digital features to facilitate understanding of the article. You can access the digital features on the article's associated Figshare page. To view digital features for this article go to https://doi.org/10.6084/m9. figshare.12821228.

\section{INTRODUCTION}

Hyperhidrosis is characterized by excessive sweating beyond that which is physiologically indicated to maintain normal thermal homeostasis [1]. In hyperhidrosis, this excessive sweating occurs regardless of environmental temperature [2] and is frequently accompanied by concomitant social and emotional stress [3]. Hyperhidrosis is caused by the overactivation of cholinergic receptors responsible for sweat gland activation [4], and sweat production among patients with hyperhidrosis is about four- to fivefold greater than that of individuals without the condition [5].

Primary hyperhidrosis is idiopathic and usually multi-focal [2] and results from overactivity of the sympathetic nerves. The most frequently affected areas are the axillae, the palms of the hands, soles of feet, and craniofacial regions [6, 7]. Diagnostic criteria for primary focal hyperhidrosis includes focal, visible, and excessive sweating of at least 6 months' duration without apparent cause, in addition to at least two of the following characteristics: sweating is (1) bilateral and symmetric, (2) impairs daily activities, (3) occurs at least once per week, (4) has onset before 25 years of age, 
(5) is associated with a family history of primary idiopathic hyperhidrosis, and (6) typically ceases during sleep [8]. Secondary hyperhidrosis is excessive sweating that is attributable to underlying medical conditions or medications.

The prevalence of hyperhidrosis in the USA, including those who may be undiagnosed, is estimated at $4.8 \%$, or 15.3 million affected individuals [2]. However, patients may be underdiagnosed and undertreated, and they may not seek treatment for their condition. For example, one study conducted among 393 patients with probable hyperhidrosis reported that only about half of respondents stated that they discussed their excessive sweating with a healthcare professional [2]. Thus, diagnosed prevalence rates based on retrospective administrative claims studies are much lower than the overall prevalence estimate cited above, and range from 0.1 to $0.4 \%[9,10]$.

First-line treatment recommendations for primary focal axillary hyperhidrosis include a topical antiperspirant that may be either a clinical-strength formulation available over the counter or a prescription-strength formulation containing aluminum and zirconium salts, or glycopyrronium cloth, which was approved in June 2018 and made commercially available in October 2018 for treatment of primary axillary hyperhidrosis in the USA $[11,12]$. Second-line therapies include focal injections of botulinum toxin A or microwave thermolysis, although botulinum toxin A may be used as a first-line therapy in severe cases [13]. Other options for persistent symptoms of hyperhidrosis include oral systemic therapies, such as glycopyrrolate or oxybutynin, removal of sweat glands (suction curettage), and endoscopic thoracic sympathectomy surgery [11].

Hyperhidrosis may induce stress and impact individuals' social and professional lives, and may be associated with a reduced quality of life and mental health issues. Patients report a significant quality of life impairment that impacts all aspects of their lives, including social embarrassment, interference with daily living activities, employment, and intimacy $[3,13,14]$. To date, limited information is available on the impact of hyperhidrosis on mental health conditions, such as depression and anxiety, from observational studies conducted in real-world settings. A 2019 systematic review identified seven studies which examined the association between primary hyperhidrosis and anxiety and depression, with the authors concluding that there is a dearth of research describing the psychological impact of hyperhidrosis [15]. A 2016 case-control study found higher adjusted odds of anxiety [odds ratio (OR) 3.25, 95\% confidence interval (CI) 2.40-4.50] and depression (OR 3.23, 95\% CI 2.40-4.30) among patients with hyperhidrosis compared to those without, with the adjusted odds of anxiety and depression increasing with hyperhidrosis severity [16]. A 2014 cross-sectional study of patients with severe primary hyperhidrosis in Brazil estimated that almost half $(49.6 \%)$ had anxiety, while $11.2 \%$ had depression, suggesting a significant mental health burden among persons with hyperhidrosis [17]. However, the real-world rates of diagnosed anxiety and depression have not been evaluated among a large, nationally representative cohort of hyperhidrosis patients in the USA. Additional research is warranted to characterize the association between mental health and hyperhidrosis among larger patient populations, as well as to characterize current treatment patterns in usual-care settings.

The objective of this study was to describe both patient and treatment characteristics and to estimate the prevalence of depression and anxiety among patients with hyperhidrosis in the USA. Secondary objectives were to identify patient factors associated with hyperhidrosis treatment, and incident depression and/or anxiety, among patients with hyperhidrosis.

\section{METHODS}

\section{Data Source}

This retrospective study used administrative claims data and linked enrollment information from 1 January 2009 through 30 November 2018 obtained from the Optum Research Database (ORD), a de-identified research database that contains medical and pharmacy claims data for commercial and Medicare Advantage (a 
government plan administered by private-sector health insurers) health plan enrollees in the USA. Since the patient-level data used for this study were de-identified, Institutional Review Board approval was not required for this study.

\section{Study Inclusion Criteria}

Inclusion criteria for a hyperhidrosis patient comprised at least one of the following two criteria between 1 January 2010 and 30 November 2017 (identification period): (1) a prescription claim for a prescription-strength antiperspirant' (2) an International Classification of Diseases and Related Health Problems (ICD), ninth revision (-9) or tenth revision (-10) diagnosis code for hyperhidrosis in any position from a medical claim (ICD-9: 780.8, 705.21,
705.22; ICD-10: R61, L74510, L74511, L74512, L74513, L74519, L7452) (Fig. 1). The index date was defined as the earliest qualifying encounter (ICD code or prescription claim) during the identification period. Cohort patients were required to have at least 24 months of continuous health plan enrollment. The required 12 months of continuous enrollment prior to the index date was defined as the baseline period, which was used to identify patient characteristics at the time of study initiation. Cohort patients were also required to have at least 12 months of continuous enrollment subsequent to the index date in order to enable the assessment of clinical characteristics and treatment patterns during the study follow-up period. The study follow-up period for each patient, which was at least 12 months in duration starting on the index date, ended at the

$\geq 1$ of the following: A prescription claim for a prescription-strength antiperspirant or an ICD-9 or ICD-10 diagnosis code for hyperhidrosis in any position between January 1, 2010- November 30, 2017 $(n=309,709)$

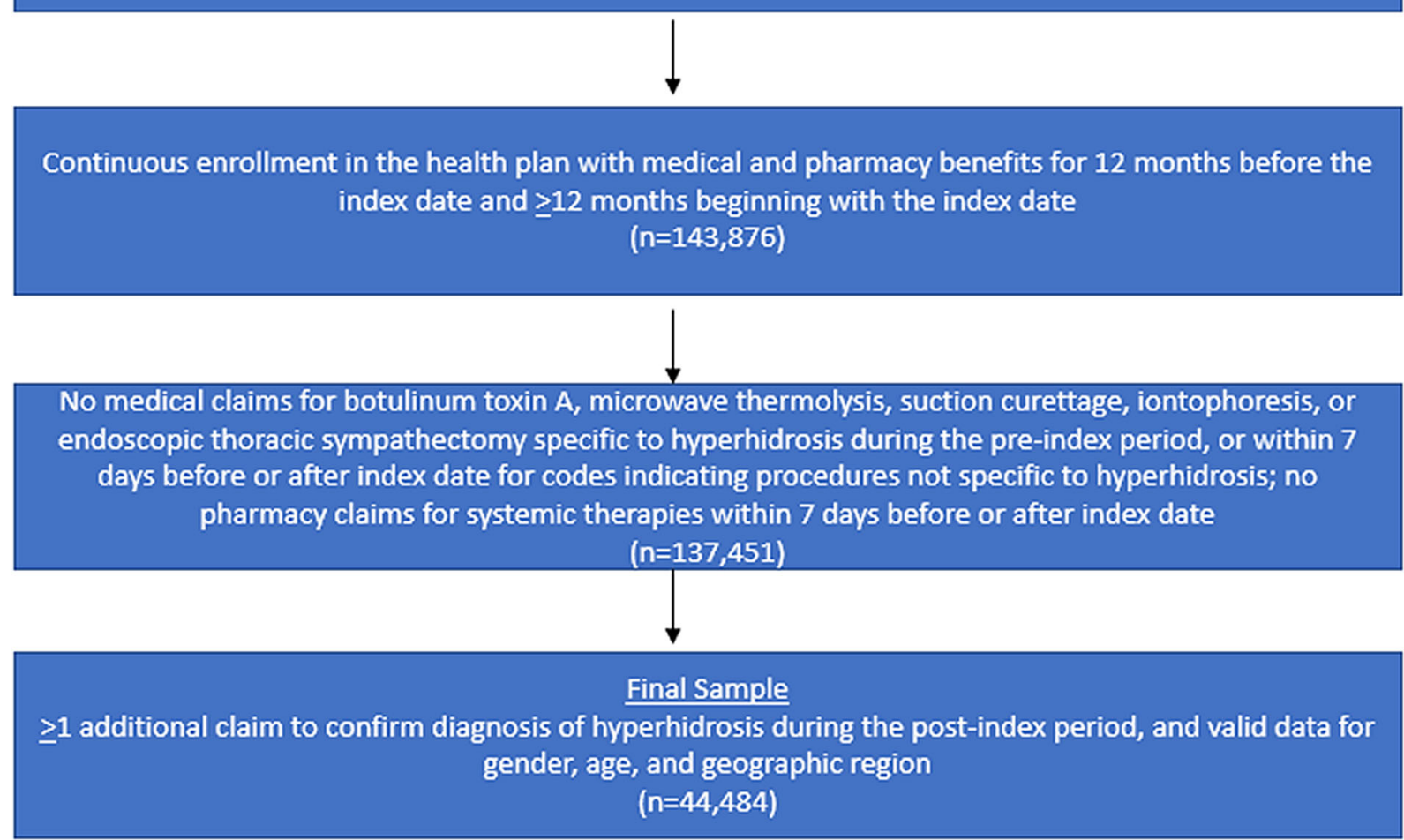

Fig. 1 Flowchart of hyperhidrosis patient selection. ICD-9/-10 International Statistical Classification of Diseases and Related Health Problems, 9th/10th revision 
earlier of disenrollment or the end of the study period. This variable follow-up period was used to analyze measures specific to patients with hyperhidrosis and constituted a preliminary 'non-confirmatory hyperhidrosis' patient cohort $(n=143,876)$.

To construct a 'confirmatory hyperhidrosis' patient cohort, at least one additional prescription claim or diagnosis code during the followup period was required. This confirmatory hyperhidrosis cohort also required both of the following: (1) no medical claims for botulinum toxin A, microwave thermolysis, suction curettage, iontophoresis, or endoscopic thoracic sympathectomy during the pre-index period, for codes indicating procedures specific to the treatment of hyperhidrosis, and (2) no medical claims for botulinum toxin $\mathrm{A}$, microwave thermolysis, suction curettage, iontophoresis, or endoscopic thoracic sympathectomy, and no pharmacy claims for oral systemic therapies, within 7 days preceding/following the index date, for codes indicating procedures or medications not specific to the treatment of hyperhidrosis (Fig. 1). For patients whose index date was in 2010 (i.e., the first year of the observation period), evidence of hyperhidrosis was further sought in 2009 in order to ensure that a code for a procedure or medication not specific to the treatment of hyperhidrosis was not present within 7 days of such evidence. Finally, eligible cohort patients were required to have valid data for gender, age, and geographic region in the ORD (Fig. 1).

A control cohort of patients without hyperhidrosis was used to compare characteristics to patients with hyperhidrosis. Control cohort patient inclusion criteria included the following: (1) no evidence of hyperhidrosis (medical claims with diagnoses of hyperhidrosis or pharmacy claims for prescription-strength antiperspirant), and (2) the same continuous enrollment criteria as the hyperhidrosis cohort. For each patient, all dates corresponding to healthcare resource utilization (e.g., a claim for an office visit, inpatient admission, or prescription drug) during the identification period were identified; subsequently, the index date for control cohort patients was determined by random selection of one healthcare resource utilization date. Control cohort patients were categorically matched 1:1 to non-confirmatory hyperhidrosis cohort patients based on index year, age, gender, and healthcare plan region $(n=143,876)$. These patients were followed for 12 months starting on the index date, and continuous enrollment in their 12 -month preand post-index periods and valid data in the fields of gender, age, and geographic region were required for inclusion. Study analyses were conducted on the 'confirmatory hyperhidrosis' cohort and all controls identified via matching to the 'non-confirmatory' hyperhidrosis cohort, and a uniform 12-month follow-up period was used for the comparative analyses of the hyperhidrosis and control cohorts.

\section{Study Measures}

Primary outcome variables included any treatment for hyperhidrosis (hyperhidrosis cohort only; variable follow-up period) and a diagnosis of depression, anxiety, or either depression or anxiety (hyperhidrosis and control cohorts; 12-month follow-up period). Any treatment for hyperhidrosis during the follow-up period was determined by claims history during the variable follow-up period, as indicated by prescriptionstrength antiperspirant, botulinum toxin A injection, oral systemic therapy, iontophoresis, surgical interventions (microwave thermolysis, suction curettage, endoscopic thoracic sympathectomy), and/or glycopyrronium cloth. A depression diagnosis was determined based on $\geq$ 1 medical claim with a diagnosis code for depression in any diagnostic position and/or $\geq 1$ pharmacy claim for a medication indicated only as an antidepressant [Electronic Supplementary Material (ESM) 1]. A second indicator variable identified patients with new depression; i.e., depression that was not identified during the pre-index period. Similarly, anxiety was determined based on $\geq 1$ medical claim with a diagnosis code for anxiety in any diagnostic position and/or $\geq 1$ pharmacy claim for a medication indicated only as an anxiolytic (ESM 1). A second indicator variable identified patients with incident anxiety. The presence of depression and/or anxiety was also identified as defined above 
while also including patients without a diagnosis code for depression or anxiety but with $\geq 1$ pharmacy claim for a medication indicated for the treatment of both depression and anxiety (ESM 1). A separate indicator variable identified patients with incident depression or anxiety; i.e., depression or anxiety that was not identified during the baseline period. Depression, anxiety, and depression and/or anxiety were identified at baseline and during the 12-month follow-up for the hyperhidrosis and control groups.

Secondary outcome variables included proportion of days covered (PDC), which is a measure of adherence to chronic medications that has been used for dermatologic conditions in other studies (hyperhidrosis cohort only; 12-month follow-up period) [18]. PDC was used to estimate patient medication adherence for prescription-strength antiperspirants and systemic therapies only, since other treatment regimens did not involve a quantifiable patient adherence component to therapy. PDC was calculated for the first 12 months of follow-up by dividing the number of days on which medication was available based on filled prescriptions by the number of days between the earliest prescription claim in the observation period through the end of the observation period (i.e., 12 months of follow-up). PDC was measured both as a continuous variable (i.e., percentage of time during the follow-up period "covered" by prescription claims) as well as the proportion of patients who were adherent, defined as PDC $\geq 0.8$ [18]. Other variables of interest included index year, age, gender, and geographic region. Baseline comorbid conditions were captured; these were defined using indicator variables for specific disease conditions based on ICD-9-CM (Clinical Modification) and ICD-10 diagnoses employed by the Clinical Classifications Software managed by the Agency for Healthcare Research and Quality (AHRQ) [19].

\section{Statistical Analysis}

All study variables were analyzed descriptively by calculating counts and percentages for categorical variables and means, standard deviations (SD), and medians for continuous variables. Occurrence, counts, and time until treatments were summarized. The number of patients with each treatment and the months to the first observed claim for treatment were counted. To account for variable follow-up in the sample, we also calculated treatments as incidence rates, with number of events (i.e., patients who had the treatment), number of person-years, and rates of treatments (events) per person-year. In addition, we performed Kaplan-Meier analysis of the time to the event for all patients, censoring for the event or end of the variable post-index period. Clinical characteristics were compared between the hyperhidrosis and control cohorts accounting for matching: $Z$ tests using robust standard errors in an ordinary least squares regression analysis were used for continuous measures; Rao-Scott tests were used for binary measures. All measures that were compared between cohorts, including mental health indicators, were based on the uniform 12-month follow-up period for these patients.

Multivariable logistic regression was used to control for possible confounding of the relationship between independent variables and outcomes. The inclusion of specific independent variables in each model was determined using clinical rationale and statistical significance $(p<0.05)$. Regression diagnostics were used to assess goodness of fit and check for model assumption violations.

\section{RESULTS}

\section{Cohort Attrition}

During the study identification period, 309,709 individuals had at least one prescription claim for a prescription-strength antiperspirant or an ICD code for hyperhidrosis (Fig. 1). The initial non-confirmatory hyperhidrosis cohort comprised 137,451 patients. Subsequent to application of additional study eligibility criteria to confirm hyperhidrosis diagnosis, the final confirmatory hyperhidrosis cohort comprised 44,484 patients who were compared to 137,451 controls without hyperhidrosis. 
Table 1 Demographic and clinical characteristics of patients in the hyperhidrosis and control groups

\begin{tabular}{lccr}
\hline $\begin{array}{l}\text { Demographic and clinical } \\
\text { characteristics }\end{array}$ & $\begin{array}{l}\text { Control group } \\
(\boldsymbol{n}=\mathbf{1 3 7}, \mathbf{4 5 1})\end{array}$ & $\begin{array}{l}\text { Hyperhidrosis group } \\
(\boldsymbol{n}=\mathbf{4 4 , 4 8 4})\end{array}$ & $\boldsymbol{p}^{\text {value }^{\mathbf{a}}}$ \\
\hline Mean age, years (SD) & $40.2(16.9)$ & $36.5(16.5)$ & $<0.001$ \\
Female, $n$ (\%) & $76,930(56.0 \%)$ & $26,028(58.5 \%)$ & $<0.001$ \\
US census region, $n$ (\%) & $15,786(11.5 \%)$ & $5517(12.4 \%)$ & $<0.001$ \\
Northeast & $42,254(30.7 \%)$ & $13,522(30.4 \%)$ & 0.056 \\
Midwest & $57,056(41.5 \%)$ & $17,843(40.1 \%)$ & $<0.001$ \\
South & $22,322(16.2 \%)$ & $7595(17.1 \%)$ & $<0.001$ \\
West & $33(0.0 \%)$ & $7(0.0 \%)$ & 0.205 \\
Other & $42,227(30.7 \%)$ & $17,330(39.0 \%)$ & $<0.001$ \\
Comorbid conditions, $n$ (\%) & $8840(19.9 \%)$ & $<0.001$ \\
Respiratory infections & $20,785(15.1 \%)$ & $8029(18.1 \%)$ & $<0.001$ \\
Other lower respiratory infections & $19,394(14.1 \%)$ & $6310(14.2 \%)$ & $<0.001$ \\
Other upper respiratory infections & $13,438(9.8 \%)$ & $13,734(30.9 \%)$ & $<0.001$ \\
Other gastrointestinal disorders & $28,187(20.5 \%)$ & $12,745(28.7 \%)$ & $<0.001$ \\
Other skin disorders & $33,509(24.4 \%)$ & & \\
Other connective tissue disorders & & & \\
\hline
\end{tabular}

$S D$ Standard deviation

${ }^{a} p$ values were computed with clustering, with the $Z$ test using robust standard errors in an ordinary least squares regression analysis for continuous measures, and the Rao-Scott test for binary measures

b A total of 172 skin conditions were identified in this category that applied to this patient sample. The most common condition was "other acne" ( $n=4025$, or $29.3 \%$ of patients identified with "other skin disorders"); diagnosis codes referring to acne were identified in 4865 patients, or $35.4 \%$ of patients identified with "other skin disorders"

\section{Patient Characteristics}

The mean age $( \pm$ SD) of patients in the hyperhidrosis cohort was $36.5( \pm 16.5)$ years (Table 1$)$ and that of the control cohort was $40.2( \pm 16.9)$ years $(p<0.001)$. Fewer than $1 \%$ of hyperhidrosis patients were aged $\leq 8$ years, and $15.7 \%$ were aged 9-17 years (Fig. 2). The hyperhidrosis cohort and control cohorts were predominantly female (58.5 and 56.0\%, respectively) $(p<0.001)$ (Table 1). The highest proportion of study patients were from the Southern region of the USA, consistent with the geographic distribution of enrollment. Comorbid conditions were more prevalent in the hyperhidrosis cohort than among controls (all $p<0.001$ ). The

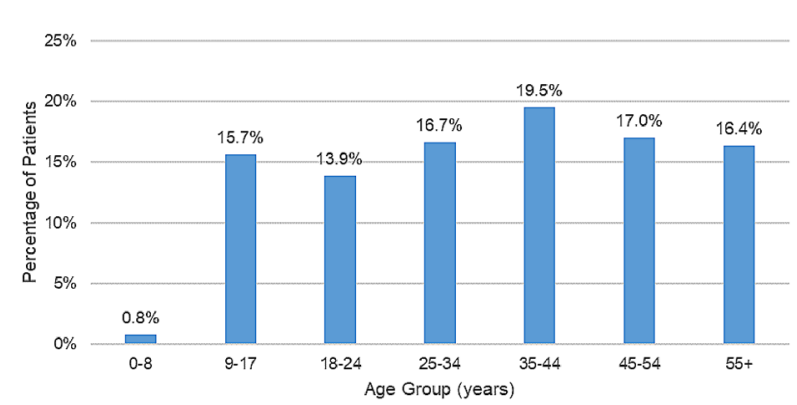

Fig. 2 Age distribution of hyperhidrosis cohort

most common comorbid conditions among hyperhidrosis patients were respiratory infections $(39.0 \%)$, other skin disorders (30.9\%), and other connective tissue disorders $(28.7 \%)$. Of 
A 60

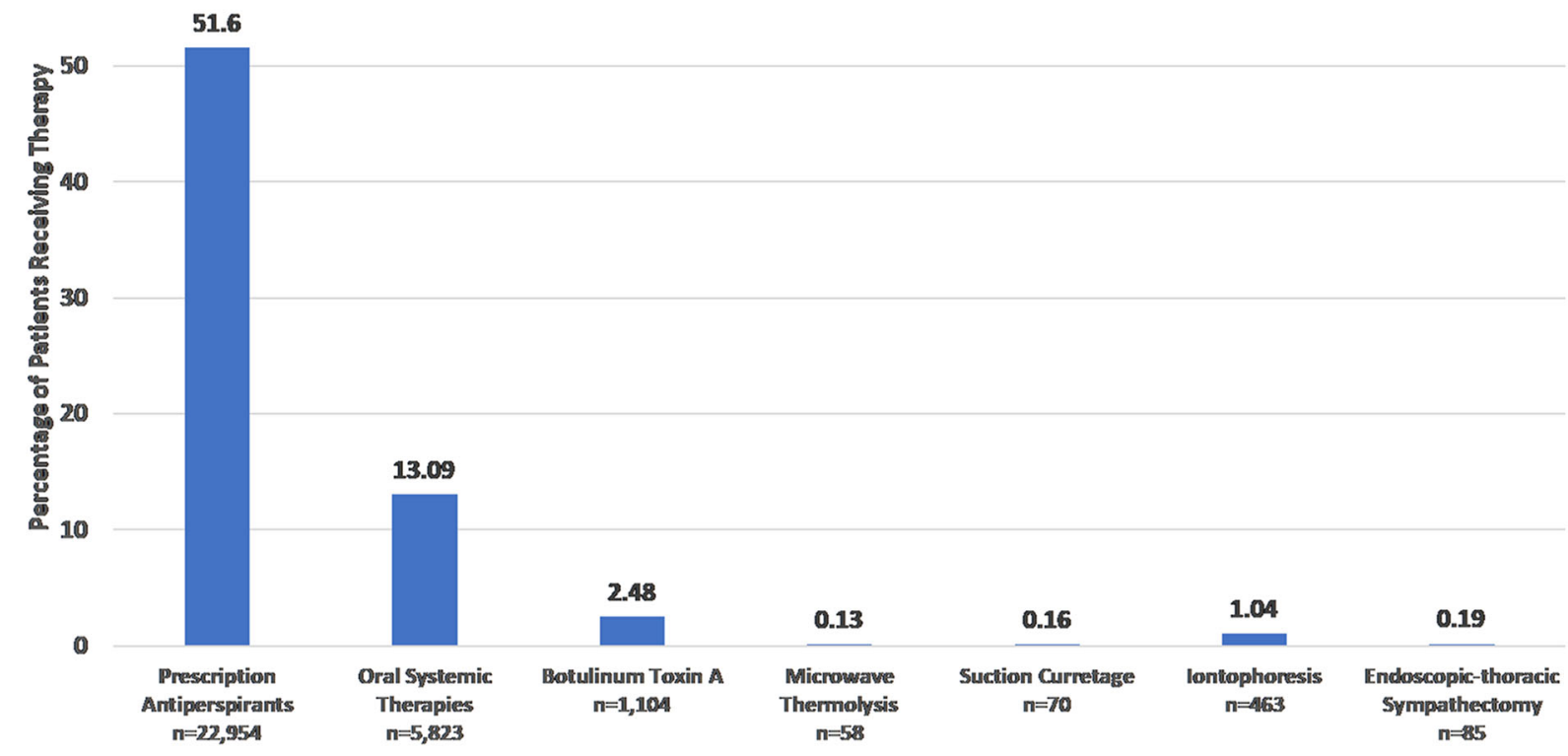

B

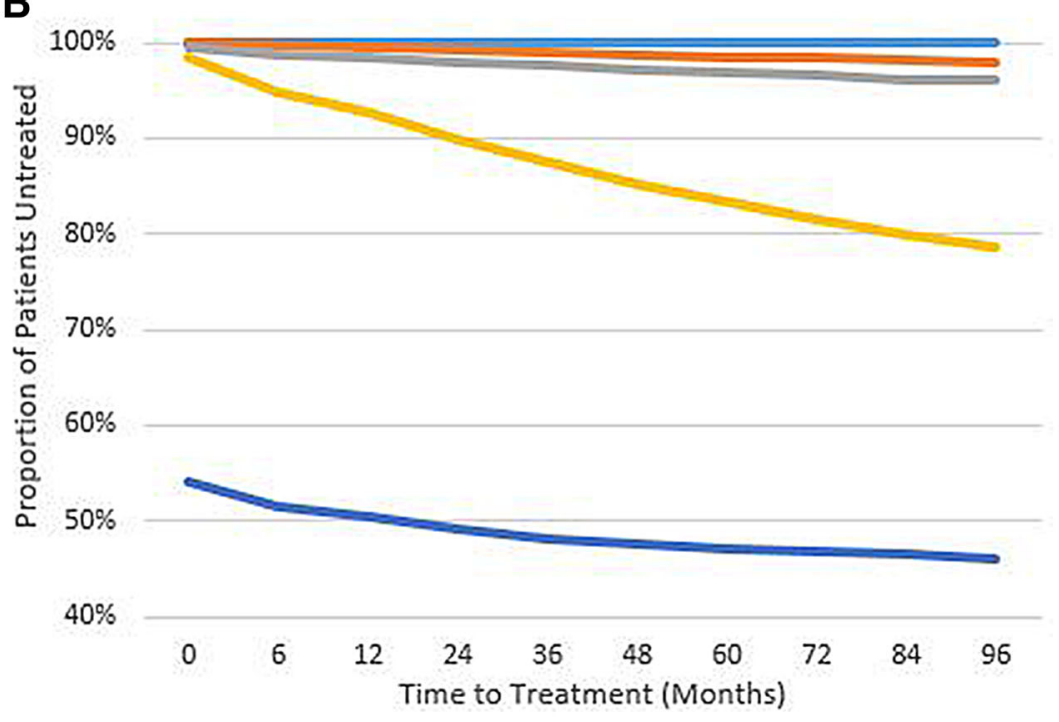

$\Longrightarrow$ Endoscopic thoracic sympathectomy

lontophoresis

$\Longrightarrow$ Botulinum Toxin A Injection

$\Longrightarrow$ Oral Systemic Therapies

Extra-or Prescription- Strength Antiperspirant

Fig. 3 Overall proportion of patients receiving treatments and procedures in the hyperhidrosis cohort (a) and Kaplan-Meier analysis of adjusted time to treatments and procedures (b). a Percentages do not take variable follow-up period into account. b Glycopyrronium cloth,

note, the three most common ICD diagnosis codes for other skin disorders included acne (9\%), rash and other non-specific skin eruption (3\%), and actinic keratosis (3\%). microwave thermolysis, and suction curretage were also measured, but as very few patients had these treatments, their trend lines are not shown. The log-rank test was used for assessing equality of survival distributions

More than half of hyperhidrosis patients were treated with a prescription-strength antiperspirant within 12 months of the index date $(51.6 \%), 13.1 \%$ were treated with oral 
Table 2 Therapies and procedures initiated by the hyperhidrosis cohort $(n=44,484)$

\begin{tabular}{|c|c|c|c|c|c|}
\hline \multirow[t]{2}{*}{ Therapy or procedure } & \multirow{2}{*}{$\begin{array}{l}\text { Time (in months) } \\
\text { to first occurrence } \\
\text { of therapy or procedure, } \\
\text { mean }(\mathrm{SD})^{\mathrm{a}}\end{array}$} & \multicolumn{3}{|c|}{ Incidence rates } & \multirow{2}{*}{$\begin{array}{l}\text { PDC }^{\mathbf{b}}, \\
\text { mean }(S D)\end{array}$} \\
\hline & & Events & Person-years & $\begin{array}{l}\text { Rate per } \\
\text { person-years }\end{array}$ & \\
\hline $\begin{array}{l}\text { Prescription-strength antiperspirants } \\
\qquad(n=22,954)\end{array}$ & $1.4(6.3)$ & 57,756 & 83,472 & 0.69 & $0.13(0.09)$ \\
\hline Glycopyrronium cloth $(n=6)^{c}$ & $28.7(27.8)$ & 6 & 15 & 0.40 & \\
\hline Oral systemic therapies $(n=5823)$ & $16.9(19.1)$ & 40,965 & 22,709 & 1.80 & $0.30(0.28)$ \\
\hline Botulinum toxin A $(n=1104)$ & $15.1(19.5)$ & 3683 & 4530 & 0.81 & \\
\hline Microwave thermolysis $(n=58)$ & $19.9(17.8)$ & 379 & 239 & 1.59 & \\
\hline Suction curretage $(n=70)$ & $19.7(18.2)$ & 105 & 279 & 0.38 & \\
\hline Iontophoresis $(n=463)$ & $22.9(20.8)$ & 1733 & 2097 & 0.83 & \\
\hline $\begin{array}{l}\text { Endoscopic thoracic sympathectomy } \\
\qquad(n=85)\end{array}$ & $15.0(21.8)$ & 98 & 338 & 0.29 & \\
\hline
\end{tabular}

systemic therapies, $2.5 \%$ with botulinum toxin A, and $\leq 1 \%$ with iontophoresis, microwave thermolysis, suction curettage, or endoscopicthoracic sympathectomy (Fig. 3a). Kaplan-Meier analysis of adjusted time to treatments and procedures confirmed that a low proportion of patients received procedures to treat hyperhidrosis and that a substantial period of time elapsed before patients eventually received treatment and procedures (Fig. 3b; ESM 2). In the variable follow-up period, prescriptionstrength antiperspirants were initiated earliest among all treatments (Table 2), with a mean time $( \pm \mathrm{SD})$ from index date of $1.4( \pm 6.3)$ months. Other therapies and procedures were not initiated, on average, until $>1$ year following the study index date (range of means 15.0-28.7 months). Mean time to initiation for oral systemic therapies was 16.9 $( \pm 19.1)$ months. Incidence rates adjusted for the variable follow-up period ranged from 0.29 (endoscopic thoracic sympathectomy) to 1.80 (oral systemic therapies) per person-year. Medication adherence, as measured by PDC, was low for oral systemic therapies (mean $0.30, \mathrm{SD} \pm$ 0.28 ) and prescription-strength anti-perspirants (mean 0.13, SD \pm 0.09 ) (Table 2).

The results of a multivariable logistic regression model which estimated adjusted odds of receiving any treatment among the hyperhidrosis cohort are presented in Table 3 . The predictor with the largest odds of whether a patient sought any treatment for hyperhidrosis was patient age; the odds of receiving treatment was notably higher among younger patients relative to the highest age category (age $\geq 55$ years), and the odds of receiving treatment decreased steadily in each age category. Depression and anxiety at baseline were associated with $23 \%$ lower odds ratio of receiving treatment (OR 0.77, 95\% CI 0.73-0.80). Females were slightly more likely to receive treatment than males (OR 1.15, 95\% CI 1.10-1.20). Other significant predictors included presence of selected comorbid conditions; respiratory infections and gastrointestinal disorders were associated with reduced odds of treatment, 
Table 3 Logistic regression models: adjusted odds of hyperhidrosis treatment

\begin{tabular}{llll}
\hline & Odds ratio & $95 \%$ Confidence interval & $p$ value \\
\hline $\begin{array}{l}\text { Dependent variable: hyperhidrosis treatment }{ }^{\mathrm{a}} \\
\text { Independent variables }\end{array}$ & & & \\
Baseline depression or anxiety & 0.77 & $0.73-0.80$ & $<0.001$ \\
AHRQ comorbidities & & & \\
Respiratory infections & 0.93 & $0.89-0.98$ & 0.003 \\
Other lower respiratory infections & 0.64 & $0.61-0.68$ & $<0.001$ \\
Other gastrointestinal disorders & 0.59 & $0.55-0.62$ & $<0.001$ \\
Other skin conditions & 1.21 & $1.15-1.27$ & $<0.001$ \\
Female gender (reference: male) & 1.15 & $1.10-1.20$ & $<0.001$ \\
Age (years) & & & $<0.001$ \\
$<9$ & 2.23 & $1.76-2.82$ & $<0.001$ \\
$9-17$ & 18.05 & $16.51-19.73$ & $<0.001$ \\
$18-24$ & 13.44 & $12.33-14.65$ & $<0.001$ \\
$25-34$ & 8.04 & $7.42-8.71$ & $<0.001$ \\
$35-44$ & 4.54 & $4.21-4.91$ & $<0.001$ \\
$45-54$ & 2.08 & $1.92-2.25$ & - \\
$\geq 55$ & Reference & & \\
\hline
\end{tabular}

${ }^{a}$ Regression model was fitted for hyperhidrosis cohort only. Number of observations used in regression model $=44,484$

while other skin conditions were associated with greater odds of treatment (Table 3).

The proportion of patients in the hyperhidrosis and control cohorts with either anxiety or depression at study baseline and during follow-up are shown in Fig. 4. The proportion of patients in the hyperhidrosis cohort with depression, anxiety, and either anxiety or depression during the 12-month follow-up period were $21.6 \%$ (vs. $14.3 \%$ for controls), $27.8 \%$ (17.4\% for controls), and 41.1\% (28.2\% for controls), respectively (all $p<0.001$ vs. control cohort). The proportion of hyperhidrosis patients with new depression, anxiety, and either anxiety or depression, which includes only the indicators of each measure identified during the follow-up period, were 10.2, 15.2, and $18.2 \%$, respectively, compared to the control cohort $(5.9,8.5$, and $10.6 \%$, respectively) (all $p<0.001$ vs. control cohort). Multivariable logistic regression was used to estimate adjusted odds of depression and/or anxiety among both hyperhidrosis and control cohorts (Table 4). This analysis revealed that the adjusted odds of depression and/or anxiety were $76 \%$ greater for the hyperhidrosis cohort (OR 1.76, 95\% CI 1.72-1.80) as compared to the controls. Other significant predictors included patient age, female gender, and presence of comorbid conditions. The oldest age group was more likely to experience depression and/or anxiety, and odds of depression/anxiety decreased with decreasing age. Patients with gastrointestinal disorders had increased odds of experiencing depression/ anxiety (OR 1.82, 95\% CI 1.77-1.88). 


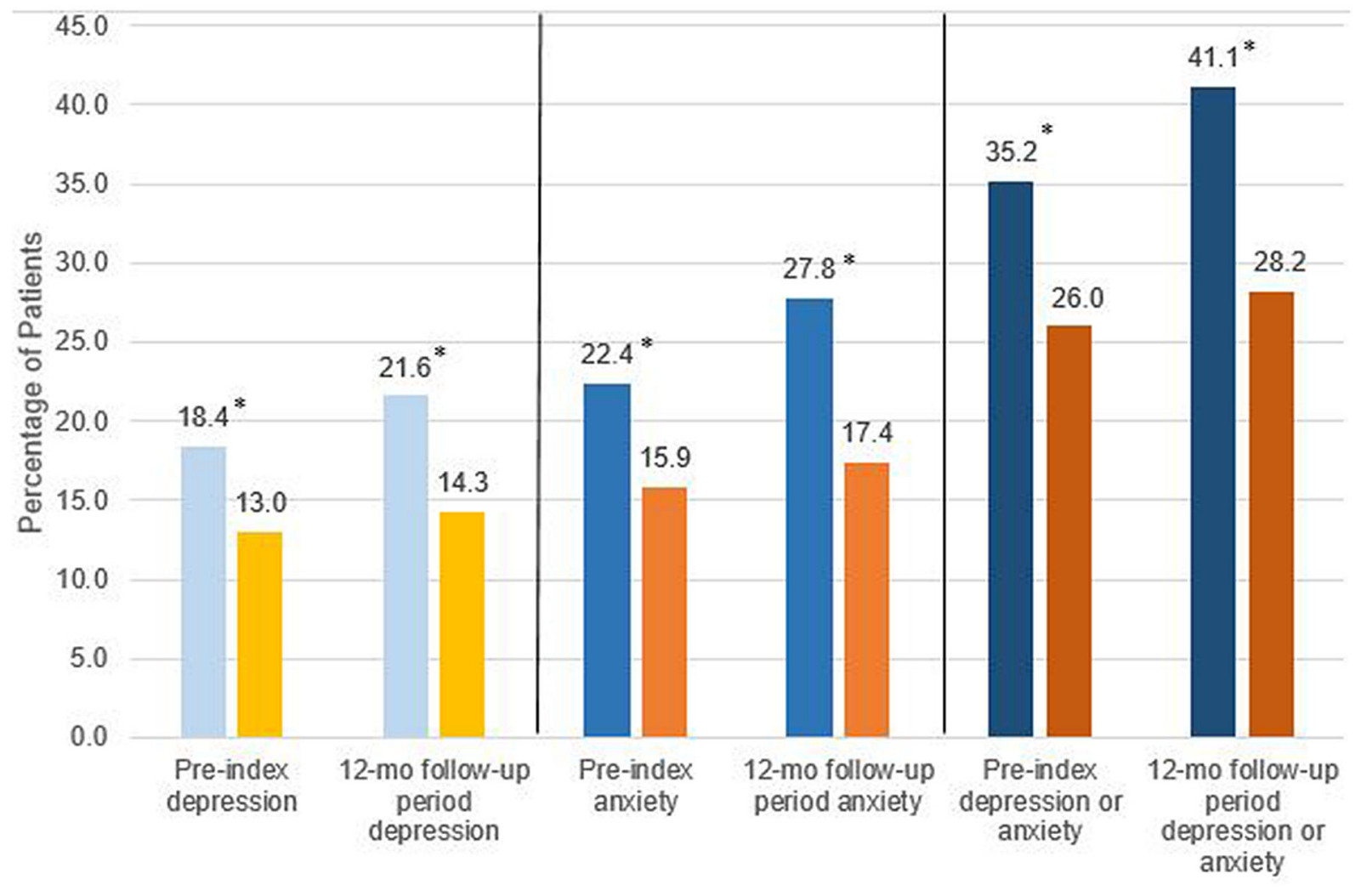

Fig. 4 Pre- and post-index (total observation period) depression and anxiety in the hyperhidrosis and control groups. Blue bars represent the hyperhidrosis cohort;

\section{DISCUSSION}

This retrospective study of hyperhidrosis in usual-care settings found both low rates of treatment for hyperhidrosis and high rates of concomitant depression and/or anxiety among these patients. Many of the patients with hyperhidrosis included in this study did not receive treatment within $\geq 12$ months of follow -up following a new hyperhidrosis diagnosis; during the variable follow-up period, only $52 \%$ of the hyperhidrosis cohort had a prescription filled for prescription-strength antiperspirants, the most common hyperhidrosis treatment among this cohort. Consistent with prior studies, we found high rates of concomitant depression and anxiety among individuals with hyperhidrosis. Compared to matched controls, a higher percentage of patients with hyperhidrosis had depression/anxiety during follow- orange bars represent the control group. *Significant difference $(p<0.001)$ compared to control group

up (41.1 vs. $28.2 \%, p<0.001$ ), which corresponded to higher adjusted odds of depression/ anxiety in patients with hyperhidrosis (OR 1.76, 95\% CI 1.72-1.80) while controlling for other factors. Patients with hyperhidrosis also had a higher percentage of newly diagnosed depression/anxiety during the 12-month follow-up period (18.2 vs. $10.6 \%, p<0.001)$.

It is estimated that each year, $19.1 \%$ of adults in the USA have had an anxiety disorder during the previous year, with higher rates for females $(23.4 \%)$ than males $(14.3 \%)$, and that an estimated $6.8 \%$ of US adults have had a major depressive disorder in the past year (females $8.6 \%$, males $4.9 \%$ ) [20]. In the current study, at baseline, 22 and $18 \%$ of patients with hyperhidrosis had anxiety and depression, respectively, and during the 12 months following hyperhidrosis diagnosis, 28 and $22 \%$ of patients had anxiety and depression, respectively. Notably, anxiety and depression prevalence in 
Table 4 Logistic regression model: adjusted odds of depression and/or anxiety

\begin{tabular}{llll}
\hline & Odds ratio & 95\% Confidence interval & $p$ value \\
\hline $\begin{array}{l}\text { Dependent variable: depression and/or anxiety, 12-month follow-up period } \\
\text { Independent variables }\end{array}$ & & \\
Hyperhidrosis cohort (reference: control cohort) & 1.76 & $1.72-1.80$ & $<0.001$ \\
Comorbid conditions & & & \\
Respiratory infections & 1.28 & $1.25-1.31$ & $<0.001$ \\
Other lower respiratory infections & 1.43 & $1.40-1.48$ & $<0.001$ \\
Other upper respiratory infections & 1.24 & $1.21-1.28$ & $<0.001$ \\
Other gastrointestinal disorders & 1.82 & $1.77-1.88$ & $<0.001$ \\
Other skin conditions & 1.20 & $1.17-1.23$ & $<0.001$ \\
Female gender (reference: male) & 1.75 & $1.71-1.79$ & $<0.001$ \\
Age (years) & & & $<0.001$ \\
$<9$ & 0.16 & $0.14-0.19$ & $<0.001$ \\
$9-17$ & 0.37 & $0.36-0.38$ & $<0.001$ \\
$18-24$ & 0.64 & $0.62-0.67$ & $<0.001$ \\
$25-34$ & 0.78 & $0.76-0.80$ & - \\
$\geq 35$ & ref & - & \\
\hline
\end{tabular}

${ }^{a}$ Regression model was fitted for hyperhidrosis and control cohorts

b Logistic model was also estimated with an indicator of a match between hyperhidrosis and control cohorts with no change to model results. Observations used $=44,484$ (hyperhidrosis); 137,451 (controls)

the current study, even at baseline, was higher than national prevalence estimates, possibly reflecting an impact of hyperhidrosis on emotional health in patients with this condition during a period in which there are symptoms but not necessarily a diagnosis or discussion of treatment with a healthcare provider. The proportion of study patients with a diagnosis of anxiety and/or depression increased substantially following the study index date, suggesting that despite the high baseline prevalence of these conditions, among some individuals, receipt of a hyperhidrosis diagnosis may have had additional impact on their emotional health and well-being. Additional research is needed to understand this association.

A significant impact on quality of life measures associated with hyperhidrosis has been documented. Hyperhidrosis is a chronic condition that has a broad impact on quality of life and has been shown to affect daily activities such as activity and situational avoidance, as well a patient's personal, professional, and social life [14]. Several studies have demonstrated a moderate to very large effect on quality of life such that active intervention is warranted, and the impact of hyperhidrosis on quality of life may be similar to or greater than that of other chronic dermatological conditions, such as psoriasis, eczema, or acne [3].

The mental and emotional health consequences of hyperhidrosis may be comparable to that often experienced by individuals with gastrointestinal (GI) disorders, which are associated with significant concomitant depression and anxiety. In a 2016 study by Zhang et al. among 
gastroenterology inpatient/outpatients with chronic GI conditions, including peptic ulcer, digestive tumors, chronic gastritis, functional dyspepsia, irritable bowel disease, and irritable bowel syndrome, $31 \%$ had symptoms of depression, $27 \%$ anxiety, and 37\% depression and/or anxiety [21]. In our study, among hyperhidrosis patients, $22 \%$ had depression, $28 \%$ had anxiety, and $41 \%$ had depression and/ or anxiety during the 12-month post-index period following the initial diagnosis of hyperhidrosis.

Limited information is currently available on the impact of hyperhidrosis on mental health and overall well-being. Braganca et al. evaluated depression and anxiety prevalence in a retrospective claims study of 197 patients who sought treatment for hyperhidrosis at a single practice in Brazil [17]. However, this study was not designed to estimate treatment rates and did not report on the types of treatment received by the study population. In addition, the study cohort was limited to those with 'severe hyperhidrosis' and treated individuals and may not include those with milder forms of hyperhidrosis. Furthermore, our study results may be more generalizable than those of Braganca and colleagues, and it is the first realworld study of the impact of hyperhidrosis on depression and anxiety conducted in the USA. In another study, Bahar and colleagues evaluated 2017 consecutive dermatology outpatients from Vancouver, British Columbia and Shanghai and observed a prevalence of anxiety and depression of 21 and 27\%, respectively, among patients with hyperhidrosis, compared to 8 and $10 \%$ among those without $(p<0.001)$ [16]. Notably, in Bahar's study, anxiety and depression diagnoses were based on questionnaires via patient self-report, and not as diagnosed or treated by a healthcare provider. Finally, LopezLopez et al. studied 100 patients from an outpatient medical center in Spain and demonstrated a relationship between hyperhidrosis and depression severity [22]. These authors showed higher Beck Depression Inventory (BDI) scores for patients with hyperhidrosis compared to those without hyperhidrosis, and that a higher severity of hyperhidrosis was associated with a higher BDI score. Of note, the mean age of the hyperhidrosis cohort of the current study (37 years) is comparable to that of Bahar et al. (mean age 36 years), while the hyperhidrosis populations studied by Braganca and LopezLopez were considerably younger (mean age of 27 years and median age of 23 years, respectively) $[16,17,22]$. Thus, it is possible that this age difference in the hyperhidrosis patients in our study was also related to hyperhidrosis clinical characteristics (such as symptom severity or duration of hyperhidrosis). Longer duration and greater severity of hyperhidrosis has been associated with anxiety and depression [22].

In the current study we found that many patients with hyperhidrosis did not receive prescription treatment after diagnosis. Given that hyperhidrosis may have an impact on health-related quality of life, this finding has important implications for the overall health and well-being of individuals with this condition. Successful treatment of hyperhidrosis may improve symptoms of depression and anxiety among affected patients. For example, in a study conducted in the USA, among patients with primary focal hyperhidrosis who underwent endoscopic thoracic sympathectomy, more than half of patients who were treated with psychotropic medications prior to surgery reduced their medication regimen, and depression and anxiety scale scores were also improved after surgery [23].

Our study has several strengths, including a large sample size and a patient cohort comprising patients from all geographic regions across the USA. Nevertheless, some limitations are important to consider when interpreting our findings. We used administrative claims to assess outpatient prescriptions filled via a pharmacy benefit, and prescriptions obtained outside the pharmacy benefit (out-of-pocket) would not be captured; this could potentially overestimate the proportion of untreated patients. Pharmacy claims capture dispensed medications and not actual medication consumption, and drug samples and over-thecounter medications, including clinical strength non-prescription antiperspirants, are not captured in this analysis. The presence of diagnosis codes used for billing purposes does 
not confirm disease; diagnosis of hyperhidrosis was obtained from ICD-9-CM and ICD-10 codes associated with a patient encounter, and due to nature of claims data, confirmation of diagnosis was not possible, nor were we able to accurately distinguish primary focal axillary hyperhidrosis from other subtypes of hyperhidrosis using ICD codes. However, this study's requirement of two hyperhidrosis claims for inclusion minimized this potential for misclassification. Procedure codes for botulinum toxin A, microwave thermolysis, suction curettage, iontophoresis, and endoscopic thoracic sympathectomy, and pharmacy codes for oral systemic therapies were non-specific to hyperhidrosis treatment. Our study design required that patients had no nonspecific codes for these procedures or pharmacy codes for oral systemic therapies within 7 days before or after the index date to address this potential limitation. However, all instances of these procedures and medications were counted in the follow-up period, which may lead to an overestimation of the frequency of these procedures related to hyperhidrosis treatment. It is important to note that while the counts and percentages of treatments and procedures utilized, as well as the mean time to first receipt of treatments and procedures during the follow-up period, provide valuable insights, the results should be interpreted with caution since they do not account for censoring. Incidence rates, along with Kaplan-Meier analyses of adjusted time to treatment, were included to address the variable follow-up period. In addition, unmeasured confounding variables may exist that could be responsible for increased rates of anxiety and depression in the hyperhidrosis cohort; however, the control cohort matching methodology was intended to minimize this. Finally, our study did not consider the potential relationship between receiving treatment for hyperhidrosis and a diagnosis of depression and/or anxiety; our analysis presents associations, not causal relationships. It is possible that patients were not offered treatment for hyperhidrosis at diagnosis (index date). A proportion of our hyperhidrosis cohort may have had secondary (rather than primary) hyperhidrosis, and thus may have been faced with a difficult primary diagnosis (e.g., cancer) with a significant impact on quality of life, or be taking a new medication for an unrelated diagnosis. Furthermore, secondary hyperhidrosis may be caused by medications that are used to treat depression. As such, additional research is warranted to understand the causal relationship between hyperhidrosis and anxiety and depression.

\section{CONCLUSION}

In this real-world observational study, hyperhidrosis was associated with an increased likelihood of depression and anxiety prior to diagnosis. We also found an increase in depression and anxiety following the diagnosis of hyperhidrosis, suggesting that some patients may experience effects on quality of life. While the current study was not designed to compare rates of depression and anxiety among those who received treatment following the diagnosis of hyperhidrosis with those who did not receive treatment, this is a pertinent area of study for future research. Hyperhidrosis may have a considerable impact on health-related quality of life which may be comparable to that associated with other chronic and impactful dermatological conditions (e.g., psoriasis, eczema) or GI disorders. Providers who treat hyperhidrosis should recognize the potential for comorbid anxiety and depression, and screening for these conditions and linking patients with mental health resources may help to improve patient overall health and well-being. Despite this comorbidity profile and the quality-of-life impact of hyperhidrosis documented in the literature, relatively small percentages of patients filled a topical or oral systemic prescription or underwent surgical treatments, suggesting that tolerability, efficacy, and provider awareness may be limiting factors in the effective treatment of patients with hyperhidrosis.

\section{ACKNOWLEDGEMENTS}

Funding. Sponsorship for this study and the journal's Rapid Service Fee were funded by 
Dermira Inc., a wholly-owned subsidiary of Eli Lilly and Company.

Authorship. All named authors meet the International Committee of Medical Journal Editors (ICMJE) criteria for authorship for this article, take responsibility for the integrity of the work as a whole, and have given their approval for this version to be published.

Medical Writing, Editorial, and Other Assistance. Medical writing and editorial assistance in the preparation of this article was provided by Jen Wogen of MedMentis Consulting LLC. Support for this assistance was funded by Optum.

Prior Presentation. Portions of this manuscript were presented as a poster at the Winter Clinical Dermatology Conference, Kohala Coast, Hawaii, 17-22 January 2020.

Disclosures. Jesse Peterson-Brandt is an employee of Optum. Kristin Khalaf Gillard is an employee of Dermira, Inc. Michael Hull was an employee of Optum at the time that that study was conducted; Michael Hull is currently employed by IQVIA. Stephanie Z. Klein is employed by Dermira as a consultant.

Compliance with Ethics Guidelines. Since the patient-level data used for this study were de-identified, Institutional Review Board approval was not required for this study.

Data Availability. The data contained in the database contains proprietary elements owned by Optum and, therefore, cannot be broadly disclosed or made publicly available. The disclosure of this data to third party clients assumes certain data security and privacy protocols are in place and that the third party client has executed our standard license agreement which includes restrictive covenants governing the use of the data.

Open Access. This article is licensed under a Creative Commons Attribution-NonCommercial 4.0 International License, which permits any non-commercial use, sharing, adaptation, distribution and reproduction in any medium or format, as long as you give appropriate credit to the original author(s) and the source, provide a link to the Creative Commons licence, and indicate if changes were made. The images or other third party material in this article are included in the article's Creative Commons licence, unless indicated otherwise in a credit line to the material. If material is not included in the article's Creative Commons licence and your intended use is not permitted by statutory regulation or exceeds the permitted use, you will need to obtain permission directly from the copyright holder. To view a copy of this licence, visit http:// creativecommons.org/licenses/by-nc/4.0/.

\section{REFERENCES}

1. Grabell DA, Hebert AA. Current and emerging medical therapies for primary hyperhidrosis. Dermatol Ther (Heidelb). 2017;7:25-36.

2. Doolittle J, Walker P, Mills T, Thurston J. Hyperhidrosis: an update on prevalence and severity in the United States. Arch Dermatol Res. 2016;308: 743-9.

3. Hamm H, Naumann MK, Kowalski JW, Kutt S, Kozma C, Teale C. Primary focal hyperhidrosis: disease characteristics and functional impairment. Dermatology. 2006;212:343-53.

4. Shibasaki M, Crandall CG. Mechanisms and controllers of eccrine sweating in humans. Front Biosci (Schol Ed). 2010;2:685-96.

5. Hund M, Kinkelin I, Naumann M, Hamm H. Definition of axillary hyperhidrosis by gravimetric assessment. Arch Dermatol. 2002;138:539-41.

6. Glaser DA, Ballard AM, Hunt NL, Pieretti LJ, Pariser DM. Prevalence of multifocal primary hyperhidrosis and symptom severity over time. Dermatol Surg. 2016;42:1347-53.

7. Estevan FA, Wolosker MB, Wolosker N, Puech-Leão P. Epidemiologic analysis of prevalence of the hyperhidrosis. An Bras Dermatol. 2017;92:630-4.

8. Hornberger J, Grimes K, Naumann M, et al. Recognition, diagnosis, and treatment of primary focal hyperhidrosis. J Am Acad Dermatol. 2004;2004(51): 274. 
9. Ricchetti-Masterson K, Symons JM, Aldridge M, et al. Epidemiology of hyperhidrosis in 2 population-based health care databases. J Am Acad Dermatol. 2018;78:358-62.

10. Mirmirani P, Carpenter DM. Skin disorders associated with obesity in children and adolescents: a population-based study. Pediatr Dermatol. 2014;31: 183-90.

11. UpToDate®. Primary focal hyperhidrosis. 2018. https://www.uptodate.com/contents/primary-focalhyperhidrosis?search=hyperhidrosis\&source=search result\&selectedTitle $=1 \sim 150$ \&usage_type $=$ default $\&$ display_rank=1. Accessed 26 Aug 2020.

12. International Hyperhidrosis Society. Hyperhidrosis treatment algorithms. 2019. https://www. sweathelp.org/treatments-hcp/clinical-guidelines/ hyperhidrosis-treatment-algorithms.html. Accessed 11 Jan 2020.

13. Solish N, Bertucci V, Dansereau A, et al. A comprehensive approach to the recognition, diagnosis, and severity-based treatment of focal hyperhidrosis: recommendations of the Canadian Hyperhidrosis Advisory Committee. Dermatol Surg. 2007;33: 908-23.

14. Kamudoni P, Mueller B, Halford J, Schouveller A, Stacey B, Salek MS. The impact of hyperhidrosis on patients' daily life and quality of life: a qualitative investigation. Health Qual Life Outcomes. 2017;15: 121.

15. Kristensen JK, Vestergaard DG, Swartling C, Bygum A. Association of primary hyperhidrosis with depression and anxiety: a systematic review. Acta Derm Venereol. 2020;100:adv00044.

16. Bahar R, Zhou P, Liu Y, et al. The prevalence of anxiety and depression in patients with or without hyperhidrosis (HH). J Am Acad Dermatol. 2016;75: 1126-33.
17. Braganca GM, Lima SO, Pinto Neto AF, Marques LM, Melo EV, Reis FP. Evaluation of anxiety and depression prevalence in patients with primary severe hyperhidrosis. An Bras Dermatol. 2014;89: 230-5.

18. Dommasch ED, Lee MP, Joyce CJ, Garry EM, Gagne JJ. Drug utilization patterns and adherence in patients on systemic medications for the treatment of psoriasis: a retrospective, comparative cohort study. J Am Acad Dermatol. 2018;79(1061-8):e1.

19. Agency for Healthcare Research and Quality. Clinical classification software (CCS) for ICD-9-CM/ ICD-10-CM. HCUP, Rockville. https://www.hcupus.ahrq.gov/toolssoftware/ccs/ccs.jsp. Accessed 26 Aug 2020.

20. Harvard Medical School. National Comorbidity Survey (NCS). Data Table 2: 12-month prevalence DSM-IV/WMH-CIDI disorders by sex and cohort. 2007. https://www.hcp.med.harvard.edu/ncs/ ftpdir/NCS-R_12-month_Prevalence_Estimatespdf. Accessed 26 Aug 2020.

21. Zhang A-Z, Wang Q-C, Huang K-M, et al. Prevalence of depression and anxiety in patients with chronic digestive system diseases: a multicenter epidemiological study. World J Gastroenterol. 2016;22:9437.

22. Lopez-Lopez D, Becerro-de-Bengoa-Vallejo R, LosaIglesias ME, Rodriguez-Sanz D, Palomo-Lopez P, Calvo-Lobo C. Relationship between depression scores and degree of skin perspiration: a novel crosssectional study. Int Wound J. 2019;16:139-43.

23. Li DC, Hulbert A, Waldbaum B, et al. Endoscopic thoracic sympathectomy for primary focal hyperhidrosis: impact on psycho-social symptomatology and psychotropic medication use. Eur J Cardiothorac Surg. 2018;54:904-11. 